\title{
Erectile function in men with end-stage liver disease improves after living donor liver transplantation
}

\author{
You-Chiuan Chien ${ }^{1 \dagger}$, Heng-Chieh Chiang ${ }^{1 \dagger}$, Ping-Yi Lin ${ }^{2}$ and Yao-Li Chen ${ }^{3,4^{*}}$
}

\begin{abstract}
Background: Impaired liver function in men can result in erectile dysfunction or hypogonadism or both. We investigated whether living donor liver transplantation (LDLT) results in improvement in male sexual function.

Methods: A total of 58 patients with end-stage liver disease (ESLD) were included in this prospective, cross-sectional study. Erectile function was measured before and after LDLT using a five-item modified version of the International Index of Erectile Function scale (IIEF-5) and hypogonadism was evaluated before and after LDLT using the Androgen Deficiency in the Aging Male (ADAM) questionnaire. Differences in mean values from the questionnaires before and after the operation were than evaluated to determine whether there is an association between LDLT and improvement in sexual function.

Results: We found that mean IIEF-5 scores significantly increased after LDLT (from $11.7 \pm 7.7$ before LDLT to $14.7 \pm 7.5$ after LDLT, $p<0.01$ ), indicating that the operation played a role in improving erectile function. In addition, the prevalence of hypogonadism among the patients with ESLD decreased markedly after liver transplantation (hypogonadism before $L D L T, n=41$ versus hypogonadism after $L D L T, n=31, p=0.03$ ). Patients with hypogonadism reported a higher prevalence of erectile dysfunction after LDLT than patients without hypogonadism $(p<0.01)$.
\end{abstract}

Conclusions: LDLT results in improvement in erectile function. In addition, improvement in erectile function is associated with self-reported absence of hypogonadism.

\section{Background}

Liver transplantation is considered standard curative therapy for patients with advanced hepatocellular carcinoma and for patients with alcohol-related end-stage liver disease (ESLD). Improvements in surgical techniques as well as medical therapy have resulted in better long-term survival outcomes for patients who require liver transplantation. However, patients with chronic disease tend to care more about quality of life than about the duration of life. Sexual function, a component of quality of life, is a major concern for men with ESLD and for those who have received liver transplantation $[1,2]$. Damage to the hypothalamic-pituitary-gonadal axis and impaired

\footnotetext{
* Correspondence: 31560@cch.org.tw

${ }^{\dagger}$ Equal contributors

${ }^{3}$ School of Medicine, Kaohsiung Medical University, Kaohsiung, Taiwan ${ }^{4}$ Department of General Surgery, Changhua Christian Hospital, No.135 Nan-Hsiao Street, Changhua county 50006, Taiwan

Full list of author information is available at the end of the article
}

liver function are the main causes of sexual dysfunction in patients with cirrhosis [1,3]. Studies have shown that orthotopic liver transplantation (OLT) can result in restoration of the hypothalamic-pituitary-gonadal axis $[4,5]$.

Religious beliefs, cultural values, educational systems and differences in legislation associated with brain death help to explain why OLT is not as common in Asian countries as in Western countries [6]. Increased need for liver transplantation and a shortage of deceased donors led to the development of living donor liver transplantation (LDLT) as an alternative to OLT. Although longterm survival rates, organ rejection rates, and rates of hepatitis $\mathrm{C}$ virus $(\mathrm{HCV})$ recurrence have been shown to be more or less equal between LDLT and OLT, studies have shown that LDLT is associated with a shorter ischemic time, a lower mortality rate, and greater perioperative benefits than OLT [7].

The prevalence of erectile dysfunction is high in patients with ESLD [8]. Although the gold standard for 
determining the presence of hypogonadism in men is measuring free testosterone level, that test is not widely performed in clinical laboratories. An alternative to measuring free testosterone in serum is to administer the selfreport Androgen Deficiency in the Aging Male (ADAM) questionnaire, which is designed to assess symptoms associated with androgen deficiency and, therefore, can be used as a tool to detect and measure the degree of hypogonadism. The ADAM questionnaire is a simple, noninvasive measure that has been shown to have a high sensitivity and a relatively acceptable specificity $[9,10]$.

Erectile dysfunction (ED) is defined as the inability to develop or maintain an erection during sexual intercourse and is the main domain of sexual dysfunction in men [8]. Although ED is an important quality-of-life issue, it is often underestimated by patients and physicians. The five-item International Index of Erection Function (IIEF-5) questionnaire provides a measurable scale with which to estimate the true prevalence of ED [11]. To the best of our knowledge, no studies have evaluated the effect of LDLT on sexual function in men. Thus, in this study, we measured the differences in pre- and post-surgical IIEF- 5 and ADAM questionnaire scores to investigate whether LDLT affects sexual function in men.

\section{Methods}

\section{Patients}

The patients in this prospective cohort study comprised 68 men with end-stage liver disease who were scheduled to undergo LDLT at the Changhua Christian hospital (Changhua, Taiwan) during the period 2006-2012. Written informed consents were obtained from the patients. The five-item International Index of Erection Function (IIEF-5) questionnaire, designed to evaluate erectile dysfunction, and the Androgen Deficiency in the Aging Male (ADAM) questionnaire, designed to measure the degree of hypogonadism, were administered to the patients before LDLT and six months after the operation. Patients were required to complete the IIEF-5 questionnaire in the outpatient department about six to seven months after transplantation. At the end of the study period, 10 men were excluded from the study because of because of working abroad $(n=2)$, because they refused to complete the questionnaires $(n=6)$, or because of self-reported inability to understand the questionnaire during the post-operative period $(n=2)$. Therefore, data on 58 patients were evaluated (Fig. 1).

The Institutional Review Board of the Changhua Christian hospital approved the study.

\section{Inclusion and exclusion criteria}

Adult ( $\geq 18$ years) male recipients of living donor liver transplant who had completed a 6-month follow-up assessment were considered eligible for inclusion. Exclusion criteria included female gender, age $<18$ years, liver graft failure, refusal to complete the post-LDLT questionnaires, and loss to follow-up for any reason.

\section{Survey}

An introductory letter comprising a detailed description of the methodology of the study, the future consequences of the study, the name of the contact person of the study, and associated information was presented to each participant before LDLT. Data collection was completed by two doctors in the urology division of the surgery department via telephone or face-to-face interview. Etiology of liver disease (hepatitis B virus, hepatitis C virus, or alcoholism),

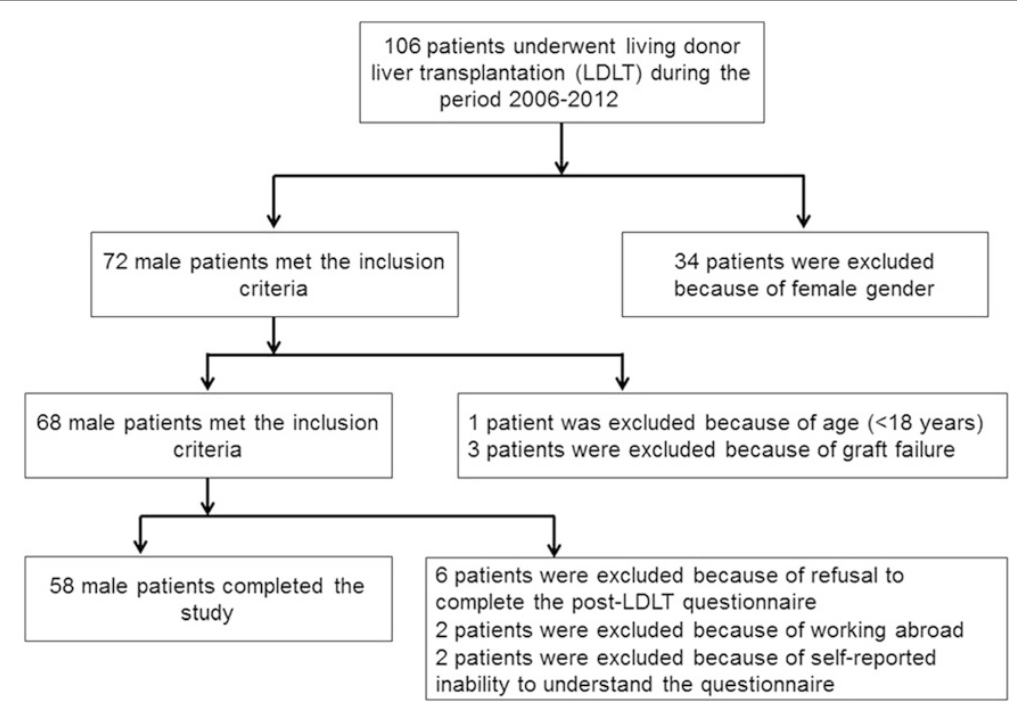

Fig. 1 Scheme of patient selection in the study 
alcohol intake history, the presence of diabetes and demographic data were obtained through chart review.

\section{Main outcome measures Measures}

The International Index of Erectile Function (IIEF), also known as the Sexual Health Inventory for Men (SHIM), is a widely used questionnaire for the evaluation of male sexual function and is recommended as a primary endpoint for diagnostic evaluation of ED severity by the National Institutes of Health Consensus Panel on Impotence [12]. The IIEF-5 is a modified version of the IIEF comprising five items instead of 15 items and was designed to shorten the time needed to complete the survey. The IIEF-5 questionnaire shows the presence and severity of ED over the past six months [13]. Studies have shown that the validity and sensitivity of the IIEF- 5 are similar to those of the original IIEF scale $[11,14]$. The IIEF- 5 is graded on a scale from 1 to 5 points for each of the five items. The total score therefore ranges from 5 to 25 . The primary score is classified into five categories of severity, namely severe (score 5-7), moderate (score 8-11), mild-to-moderate (score 12-16), mild (score 17-21), and no ED (score 22-25). The Androgen Deficiency in the Aging Male (ADAM) questionnaire is a self-report, ten-item scale designed to evaluate the degree of androgen deficiency [10]. ADAM consists of 10 questions and answering "yes" to question 1 or 7 or to 3 or more of the questions is regard as an indication of possible hypogonadal status. ADAM has been shown to be a highly sensitive but poorly specific measure for determining androgen deficiency [15]. Nonetheless, Tancredi et al. found that hormone analysis of blood samples correlated with ADAM scores [9]. Patients who reported decreased libido or inadequate erectile "strength" and patients who provided positive answers to 3 of the other questions listed in the questionnaire were considered to have androgen deficiency. Both questionnaires were given to patients before LDLT and at least six months after the operation to evaluate whether LDLT had an effect on erectile dysfunction and hypogonadism.

\section{Statistical analysis}

Categorical data were compared by the McNemar's test or the Chi-square test. Differences in continuous variables were compared by the Wilcoxon signed-rank test. A $p$ value of less than 0.05 was considered to indicate statistical significance. All statistical analyses were performed on a personal computer with the statistical package SPSS for Windows (Version 18.0, SPSS, Chicago, Il).

\section{Results}

During the period November 2012 to May 2013, 58 of the $68(85 \%)$ adult men who were eligible to participate in the study completed both questionnaires before and after LDLT. Reasons for not completing the questionnaires during the post-operative period included refusal to participate $(n=6)$, working abroad $(n=2)$, and self-reported inability to understand the questionnaire $(n=2)$. Table 1 summarizes the general characteristics of the patients who participated in the study. The mean age at the time of LDLT was $53.86 \pm 7.53$ years. Alcohol abuse was selfreported in 17 patients before LDLT and in 4 after the operation. In addition, 15 of the patients had diabetes mellitus before surgery and 27 had the disorder after LDLT (Table 1).

\section{Change in erectile dysfunction}

We found that mean IIEF-5 scores significantly increased after LDLT (from $11.7 \pm 7.7$ before LDLT to $14.7 \pm 7.5$ after LDLT, $p<0.01$ ), indicating that the operation played a role in improving erectile function. Overall, 28 patients reported improvement in erectile function, 23 patients reported no change in erectile function and 7 patients reported worse erectile function. We also found that the degrees of ED changed among the study population after surgery (Table 2). The percentage of patients with IIEF-5 scores indicative of severe ED or mild-tomoderate ED was markedly lower after LDLT (24.1\% vs. $37.9 \%$ and $17.2 \%$ vs. $31.0 \%$ respectively) and the percentage of patients with IIEF-5 scores indicating no ED was noticibly higher after LDLT ( $13.8 \%$ vs. $6.9 \%)$. Interestingly, however, the percentage of patients with scores indicative of moderate ED or mild ED was higher

Table 1 Characteristics of the population before and after transplantation

\begin{tabular}{llll}
\hline & Before transplantationN (\%) & After transplantationN (\%) & $p$ value \\
\hline Age (years; mean \pm SD) & $53.86 \pm 7.53$ & $55.02 \pm 7.33$ & $<0.001$ \\
Alcohol abuse & $17(29.3)$ & $4(6.9)$ & $<0.001$ \\
HBV & $34(58.6)$ & $1(1.7)$ & $<0.001$ \\
HCV & $17(29.3)$ & $6(10.3)$ & 0.001 \\
Diabetes & $15(25.9)$ & $27(46.6)$ & $<0.001$ \\
IIEF5 score (mean \pm SD) & $11.7 \pm 7.7$ & $14.7 \pm 7.5$ & 0.001 \\
Suspected hypogonadism via ADAM questionnaire & $41(70.6)$ & $31(53.4)$ & 0.031 \\
\hline
\end{tabular}


Table 2 Changes of erectile dysfunction prior to and after liver transplantation

\begin{tabular}{|c|c|c|c|c|}
\hline & & Before transplantationN (\%) & After transplantationN (\%) & $p$ value \\
\hline \multirow[t]{5}{*}{ Categories of IIEF5 score } & Severe ED & $22(37.9)$ & $14(24.1)$ & $<0.001$ \\
\hline & Moderate ED & $2(3.4)$ & $3(5.2)$ & \\
\hline & Mild to moderate ED & $18(31.0)$ & $10(17.2)$ & \\
\hline & Mild ED & $12(20.7)$ & $23(39.7)$ & \\
\hline & No ED & $4(6.9)$ & $8(13.8)$ & \\
\hline
\end{tabular}

after LDLT (5.2 \% vs. $3.4 \%$ and $39.7 \%$ vs. $20.7 \%$, respectively) (Table 2) (Fig. 2).

\section{Change in hypogonadism}

The prevalence of hypogonadism among the patients with ESLD decreased markedly after liver transplantation based on the scores from the ADAM questionnaires administered before and after surgery (hypogonadism before LDLT, $\mathrm{n}=41$ vs. hypogonadism after LDLT, $\mathrm{n}=31, p=0.031$ ).

\section{Association of erectile dysfunction and hypogonadism}

We found that hypogonadism before LDLT was associated with improvement in erectile function after LDLT. There was a significant correlation between hypogonadism before LDLT and IIEF-5 score prior to LDLT (Fig. 3). In patients with $\mathrm{ADAM}$ questionnaire scores indicating no hypogonadism, the mean IIEF-5 score was $15.35 \pm$ 6.89 prior to LDLT and $19.92 \pm 4.92$ after LDLT $(p<0.01)$ (Table 3). In patients with ADAM scores indicative of hypogonadism, the mean IIEF-5 score was $8.75 \pm 7.07$ before surgery and $10.53 \pm 6.65$ after LDLT $(p=0.107)$.

\section{Factors associated with post-LDLT erectile dysfunction}

The existence of HCV infection was significantly associated with lower IIEF-5 scores (mean IIEF-5 score in patients with $\mathrm{HCV}=11.06 \pm 7.81$ vs. mean IIEF-5 score in patients without $\mathrm{HCV}=16.27 \pm 6.97, p=0.015$ ). Patients with clinical evidence of DM had lower IIEF-5 scores than those without diabetes (IIEF-5 of patients with DM= $12.15 \pm 7.40$ vs. IIEF- 5 of patients without $\mathrm{DM}=17.00 \pm$
7.02, $p=0.013)$. Other parameters such as alcoholrelated origin of liver disease, development of posttransplantation DM, and gender of donor were not associated with the development of post-LDLT erectile dysfunction or hypogonadism.

\section{Discussion}

Determination of sexual health is challenging, especially in Asian men who are often reluctant to undergo evaluation of sexual function. The response rate among participants to questionnaires designed to survey sexual function has been reported to range from $22.5 \%$ to $81 \%[8,11,16]$. In our study, however, the response rate was $85 \%$. The introductory letters written by the surgeon who performed the LDLT procedures most likely contributed to the relatively high compliance rate in our study. The etiology of sexual dysfunction is multi-factorial and ESLD is one of many factors associated with the disorder. Liver transplantation restores liver function and should, theoretically, result in improvements in sexual function [8]. However, immunosuppressive drugs, which are key to survival after liver transplantation, are known to impair sexual function [17]. Alterations in the hypothalamic-pituitary-gonadal axis, changes in estrogen-over-androgen ratio, and altered sex hormone transport have been shown to cause hypogonadism in patients with impaired liver function $[16,18,19]$. Decreased testosterone level, libido, testis size and even infertility have been reported in patients with cirrhosis $[3,20]$. Foresta et al. reported decreased sex hormone binding protein levels and higher free testosterone in serum in patients after OLT, which may explain the

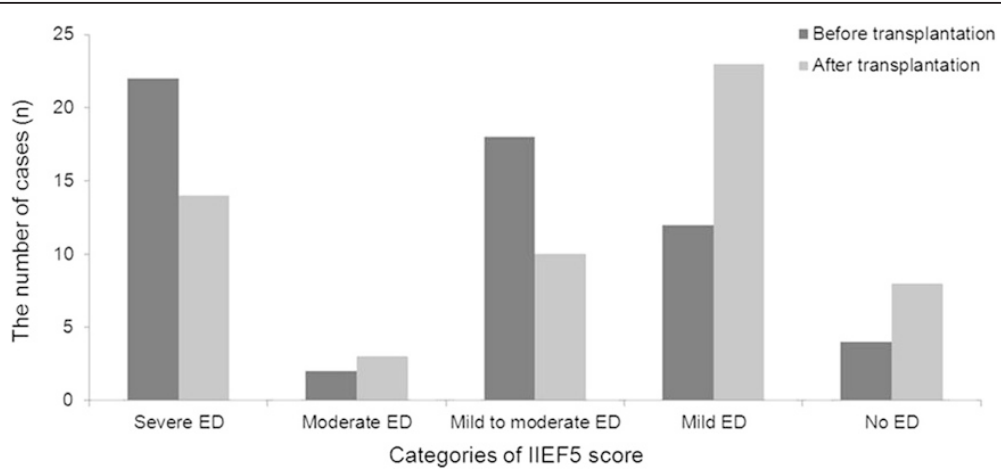

Fig. 2 Prevalence of erectile dysfunction prior to and after liver transplantation 


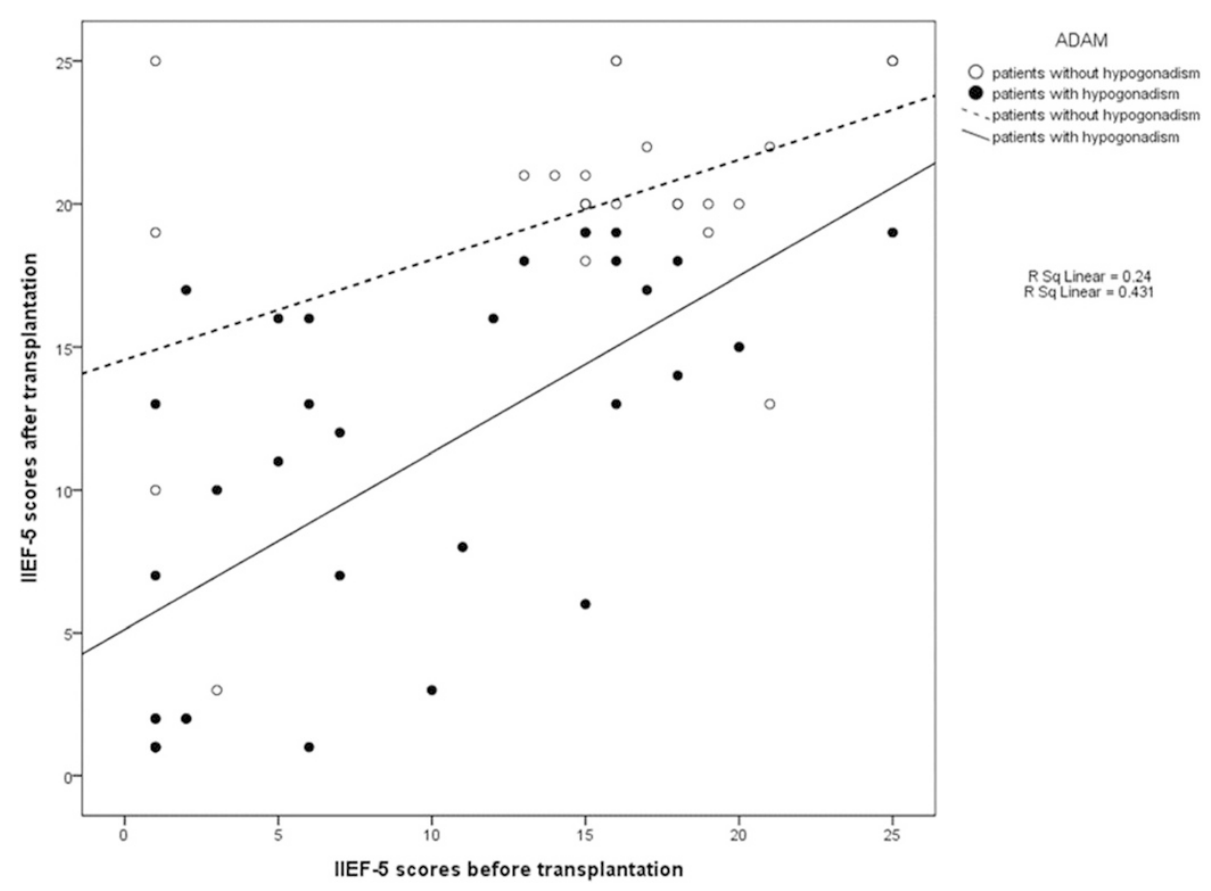

Fig. 3 Correlation between erectile dysfunction before and after transplantation for patients with or without hypogonadism

improvement in physiological function after the operation, even under immunosuppressive therapy [4]. Park et al. reported that up to $60 \%$ of men reported no improvement in erectile function after OLT [10]. Klein and colleagues reported improvement in sexual function including erectile function, sexual satisfaction and sexual desire and a statistical trend in improvement in IIEF scores after liver transplant [21]. In our study, we found that nearly half of the patients reported improvement in erectile function. Many factors contribute to ED, including drugs, neurogenic disorders, cavernosal disorders, hormones, psychological causes, surgery, aging, and diabetes [22]. In our study, higher IIEF-5 scores were associated with absence of hypogonadism after the operation. That is, improvement in erectile function after liver transplantation is mainly hormone related. Theoretically, irreversible damage of the hypothalamic-pituitary-gonadal axis by long-term consumption of alcohol implies the low possibility of improvement in sexual function after LDLT [23]. However, Huyghe E et al. reported no higher risk of erectile dysfunction in patients with end-stage liver disease secondary to alcohol consumption [8]. Burra et al. also reported that there was no significantly lower trend in IIEF score in patients with alcohol-induced ESLD [3]. Sorrell and Brown found that $40 \%$ of patients developed erectile dysfunction and $25 \%$ reported having a lower level of sexual satisfaction after liver transplantation [16]. Ho et al. reported that $32 \%$ of patients developed de novo sexual dysfunction after OLT [24]. Cardiovascular disease, posttransplantation diabetes, alcohol abuse, antidepressants and angiotensin II receptor blockers have been shown to be associated with new onset erectile dysfunction after liver transplantation [8]. In our study, $12 \%(\mathrm{n}=7)$ of our patients reported a decrease in erectile function

Table 3 IIEF-5 scores before and after transplantation for each group

\begin{tabular}{llll}
\hline & IIEF-5 score & & \\
\hline & Before transplantation(mean \pm SD) & After transplantation(mean \pm SD) & 0.109 \\
With hypogonadism & $8.75 \pm 7.07$ & $10.53 \pm 6.65$ & 0.001 \\
Without hypogonadism & $15.35 \pm 6.89$ & $19.92 \pm 4.92$ & 0.247 \\
HCV positive & $9.24 \pm 6.98$ & $11.06 \pm 7.81$ & 0.001 \\
HCV negative & $12.73 \pm 7.80$ & $16.27 \pm 6.97$ & 0.094 \\
With DM & $10.48 \pm 6.51$ & $12.15 \pm 7.40$ & 0.002 \\
Without DM & $12.77 \pm 8.53$ & $17.00 \pm 7.02$ & \\
\hline
\end{tabular}


after LDLT. The low incidence of de novo erectile dysfunction might be due to the relatively short follow-up period in our study.

There are several limitations in this study. First, immunosuppressive regimens after transplantation were not consistent throughout the study population. Therefore, we were not able to take pharmacologic factors into account. Second, our findings are limited by the relatively small sample size and short follow-up period. Third, we did not measure serum testosterone levels and therefore we do not have laboratory data to support the change in prevalence of hypogonadism. Nonetheless, to the best of our knowledge, this is the first study to compare changes in sexual function before and after LDLT. Large-scale studies with longer follow-up periods are needed to clarify the role liver transplantation plays in sexual function.

\section{Conclusions}

LDLT results in improvement in erectile function. In addition, improvement in erectile function is associated with absence of hypogonadism before LDLT.

\section{Competing interests}

The authors declare that they have no competing interests.

\section{Authors' contributions}

YCC, HCC and YLC conceptualised and planned the study. YCC writing of the manuscript, collection and analysis of data and writing of the manuscript drafts. HCC, PYL and YLC advised on study design and manuscript drafts. All authors read and approved the final manuscript.

\section{Funding}

None declared.

\begin{abstract}
Author details
'Division of urology, Department of Surgery, Changhua Christian Hospital, No.135, Nansiao St. Changhua city, Changhua county 50006, Taiwan. ${ }^{2}$ Transplant Medicine \& Surgery Research Centre, Changhua Christian Hospital, Changhua, Taiwan. ${ }^{3}$ School of Medicine, Kaohsiung Medical University, Kaohsiung, Taiwan. ${ }^{4}$ Department of General Surgery, Changhua Christian Hospital, No.135 Nan-Hsiao Street, Changhua county 50006, Taiwan.
\end{abstract}

Received: 28 December 2014 Accepted: 4 August 2015

Published online: 13 August 2015

\section{References}

1. Bravata DM, Olkin I, Barnato AE, Keeffe EB, Owens DK. Health-related quality of life after liver transplantation: a meta-analysis. Liver transplantation and surgery : official publication of the American Association for the Study of Liver Diseases and the International Liver Transplantation Society. 1999:5:318-31.

2. Durazzo M, Premoli A, Di Bisceglie C, Bo S, Ghigo E, Manieri C. Male sexual disturbances in liver diseases: what do we know? J Endocrinol Invest. 2010;33:501-5

3. Burra P, Germani G, Masier A, De Martin E, Gambato M, Salonia A, et al. Sexual dysfunction in chronic liver disease: is liver transplantation an effective cure? Transplantation. 2010;89:1425-9.

4. Foresta C, Schipilliti M, Ciarleglio FA, Lenzi A, D'Amico D. Male hypogonadism in cirrhosis and after liver transplantation. J Endocrinol Invest. 2008;31:470-8.

5. Madersbacher S, Ludvik G, Stulnig T, Grunberger T, Maier U. The impact of liver transplantation on endocrine status in men. Clin Endocrinol (Oxf). 1996;44:461-6

6. Chen CL, Kabiling CS, Concejero AM. Why does living donor liver transplantation flourish in Asia? Nat Rev Gastroenterol Hepatol. 2013;10:746-51.
7. Akamatsu N, Sugawara Y, Kokudo N. Living-donor vs deceased-donor liver transplantation for patients with hepatocellular carcinoma. World $\mathrm{j}$ hepatol. 2014;6:626-31.

8. Huyghe E, Kamar N, Wagner F, Yeung SJ, Capietto AH, El-Kahwaji L, et al. Erectile dysfunction in liver transplant patients. Am j transplant : official journal of the American Society of Transplantation and the American Society of Transplant Surgeons. 2008;8:2580-9.

9. Tancredi A, Reginster JY, Schleich F, Pire G, Maassen P, Luyckx F, et al. Interest of the androgen deficiency in aging males (ADAM) questionnaire for the identification of hypogonadism in elderly community-dwelling male volunteers. Eur j endocrinol / European Federation of Endocrine Societies. 2004;151:355-60.

10. Morley JE, Charlton E, Patrick P, Kaiser FE, Cadeau P, McCready D, et al. Validation of a screening questionnaire for androgen deficiency in aging males. Metab: clin exp. 2000;49:1239-42.

11. Wu CJ, Hsieh JT, Lin JS, Hwang TI, Jiann BP, Huang ST, et al. Comparison of prevalence between self-reported erectile dysfunction and erectile dysfunction as defined by five-item International Index of Erectile Function in Taiwanese men older than 40 years. Urology. 2007;69:743-7.

12. Rosen RC, Cappelleri JC, Gendrano 3rd N. The International Index of Erectile Function (IIEF): a state-of-the-science review. Int J Impot Res. 2002;14:226-44.

13. Rosen RC, Cappelleri JC, Smith MD, Lipsky J, Pena BM. Development and evaluation of an abridged, 5-item version of the International Index of Erectile Function (IIEF-5) as a diagnostic tool for erectile dysfunction. Int J Impot Res. 1999;11:319-26.

14. Heidelbaugh JJ. Management of erectile dysfunction. Am Fam Physician 2010;81:305-12.

15. Morley JE, Perry 3rd HM, Kevorkian RT, Patrick P. Comparison of screening questionnaires for the diagnosis of hypogonadism. Maturitas. 2006;53:424-9.

16. Sorrell JH, Brown JR. Sexual functioning in patients with end-stage liver disease before and after transplantation. Liver transpl : official publication of the American Association for the Study of Liver Diseases and the International Liver Transplantation Society. 2006;12:1473-7.

17. Rovira J, Diekmann F, Ramirez-Bajo MJ, Banon-Maneus E, Moya-Rull D, Campistol JM. Sirolimus-associated testicular toxicity: detrimental but reversible. Transplantation. 2012;93:874-9.

18. Gavaler JS, Van Thiel DH. Gonadal dysfunction and inadequate sexual performance in alcoholic cirrhotic men. Gastroenterology. 1988;95:1680-3.

19. Wang G, Yang J, Li M, Liu B, Jiang N, Fu B, et al. Liver transplant may improve erectile function in patients with benign end-stage liver disease: single-center Chinese experience. Exp clin transplant : official journal of the Middle East Society for Organ Transplantation. 2013;11:332-8.

20. Karagiannis A, Harsoulis F. Gonadal dysfunction in systemic diseases. Eur j endocrinol / European Federation of Endocrine Societies. 2005;152:501-13.

21. Klein J, Tran SN, Mentha-Dugerdil A, Giostra E, Majno P, Morard I, et al. Assessment of sexual function and conjugal satisfaction prior to and after liver transplantation. Ann transplant : quarterly of the Polish Transplantation Society. 2013;18:136-45.

22. McMahon CG, Jannini E, Waldinger M, Rowland D. Standard operating procedures in the disorders of orgasm and ejaculation. J Sex Med. 2013;10:204-29.

23. Burra P. Liver abnormalities and endocrine diseases. Best Pract Res Clin Gastroenterol. 2013;27:553-63.

24. Ho JK, Ko HH, Schaeffer DF, Erb SR, Wong C, Buczkowski AK, et al. Sexual health after orthotopic liver transplantation. Liver transpl: official publication of the American Association for the Study of Liver Diseases and the International Liver Transplantation Society. 2006;12:1478-84. 